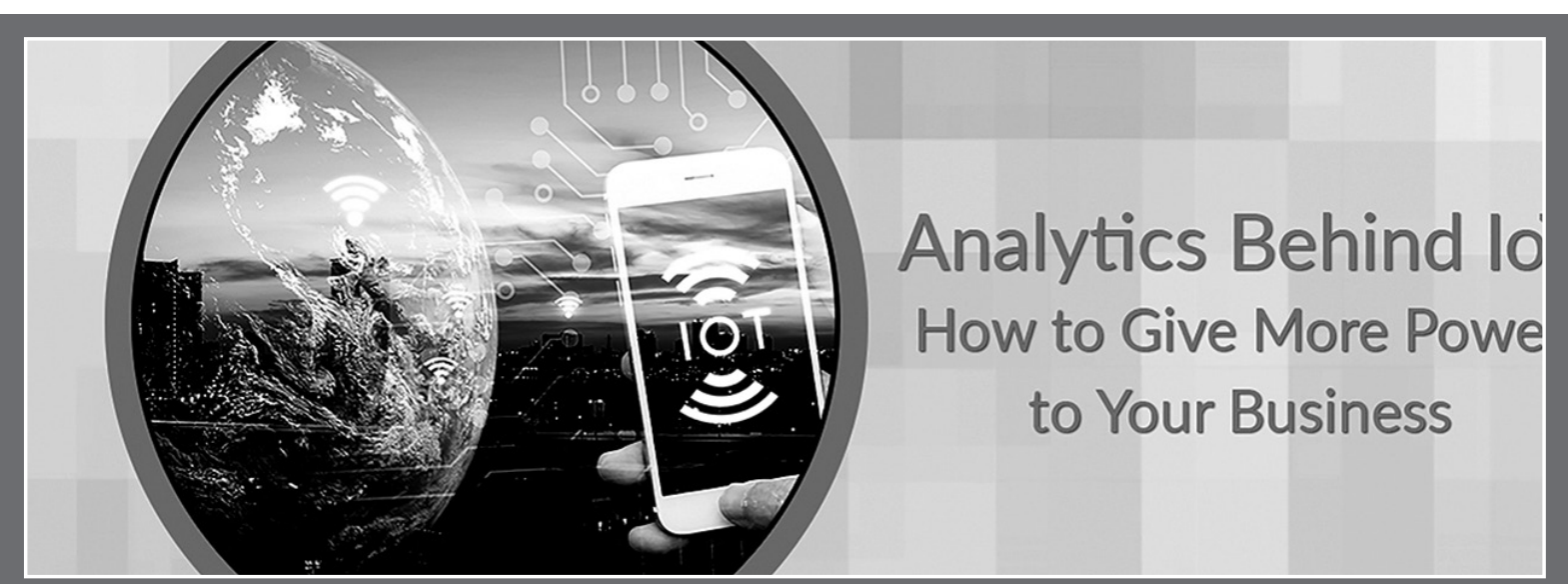

The Internet of Things (IOT): the buzzword of our times. The concept of the vast network of interconnected devices, from those in our homes to the ones in our pockets. But if we think about it, companies aren't using loT just for fun gadgets; it's actually serving many businesses and changing the way they work. loT has actually enabled a whole new world that's truly improving thanks to the amount of connectivity. For example, think of activity trackers that improve your health, or connected home electronics that reduce energy consumption. Despite how much it already helps us, how can loT go even further?

This article will explore what further possibilities can be explored by loT. We'll highlight a couple examples of businesses that have made the leap to combining advanced analytics and loT, while in the process applying it to real-time production. Hopefully, these stories inspire your company to follow in their innovative steps.

\section{Taking loT to the Next Level}

The first purpose of loT is for devices to interact with the world in order to create value, as well as to measure elements that we hadn't had the possibility to measure before. Although most of us directly think of cool gadgets like Fitbits or smart home devices, there are many developments happening within companies that seem invisible to the outside world. IoT has allowed companies to move to a new way of doing business by applying it to various processes in order to enhance productivity and efficiency. This huge amount of data can be collected and, therefore, used as a base to make new innovative decisions.

Combining IoT with advanced analytics takes it to the next level by bringing more insight on trends, and giving an opportunity for perfecting the business. Let's take a look at some case studies of companies that have made this leap:

How Can You Improve Your Soccer Team?

For a first case study, let's look over a Swiss sports company that develops wearables for athletes of various sports, in order to track and analyze performance. The way they use analytics behind their loT product consists of the following: each soccer player wears a tracking device that measures speed, sprints, acceleration and goal distance, to name just a few, during every game. These measurements are displayed in several dashboards developed with icCube, a solution that turns massive amounts of data into useful insights. Performance metrics can be compared between players, and movement is analyzed through heatmaps as an overlay on the actual soccer fields.

Several advanced calculations are made in order to see the data in various ways, therefore detecting areas for improvement. The dashboards are used by the trainer and club to help their players improve their skills, as well as to enhance tactics and strategies. This goes beyond the analytics seen on the TV during games. It aids future training approaches by carefully studying each game, as well as several historical games, at once thanks to cross analytics. 


\section{Act Fast on Issues with Live Monitoring!}

In order to better illustrate various scenarios, let's take look at a completely different example: a railroad company in the UK. The company combines IoT and operational business intelligence by using icCube to monitor train issues, in order to quickly and effectively act in case of a breakdown. The company chose icCube because, among other requirements, it has the ability to connect to on-demand custom data sources, such as the sensor data files and the MetOffice database that provides local UK temperatures. Armed with this information, employees can take immediate action in case a heating/cooling, engine or electrical issue, among many other possibilities, occurs. They can be sure they're addressing the right issue as well, thanks to reports that are automatically refreshed and directly connected to live databases. The top management department is also able to compare these values to historical data, at which point they can conduct advanced statistical analysis to evaluate if the issues could have been expected and avoided. The security of passengers is therefore highly reinforced by this real-time monitoring system.

In the digital era that we find ourselves in, we have a constant craving for technology and innovation. Therefore, companies should explore how to use the latest innovations to enhance their business. The beauty of mixing analytics and IOT is that it can be applied to the majority of industries, and for those where it still hasn't yet been applied, it'll eventually come. It's simply a matter of time and imagination.

The State of IoT Adoption in Industrial Organizations

The study outlined five levels of IOT adoption: device connectivity and data forwarding, real-time monitoring, data analytics, automation and on-board intelligence.

Seventy-eight percent of survey respondents, with transportation leading the pack, self-identified their companies at the first stage, transmitting sensor data to the cloud for analytics, and $56 \%$, again with transportation in the lead, reached the second stage, monitoring sensor data in real time for visualization.

Dave McCarthy, senior director of products at Bsquare, said he had predicted the gap between the first two stages would be smaller; no surprise there. What really surprised him, however, was the small gap between the second stage and third: Forty-eight percent of respondents said they were using data analytics for insight, predictions and optimization with applied analytics such as machine learning or artificial intelligence.

"What it indicates to me," McCarthy said, "Is that people who have gone down the visualization route have figured out, to some degree, some use of the data they're collecting, and they know that analytics is going to play a part in helping them understand more closely what that data is going to mean for them."

McCarthy wasn't surprised to see the drop in the fourth and fifth stages: Twenty-eight percent said they were automating actions across internal systems with their IoT deployments, and only $7 \%$ had reached the edge analytics level.

"Just as expected, there's a large drop-off from people doing analytics to people who are automating the results," McCarthy said. "And in my mind, the highest amount of ROI comes when you can get to those levels."

Internet of Things Gateway:

An Internet of Things (loT) gateway is a physical device or software program that serves as the connection point between the cloud and controllers, sensors and intelligent devices. All data moving to the cloud, or vice versa, goes through the gateway, which can be either a dedicated hardware appliance or software program. An loT gateway may also be referred to as an intelligent gateway or a control tier.

Some sensors generate tens of thousands of data points per second. A gateway provides a place to preprocess that data locally at the edge before sending it on to the cloud. When data is aggregated, summarized and tactically analyzed at the edge, it minimizes the volume of data that needs to be forwarded on to the cloud, which can have a big impact on response times and network transmission costs.

Another benefit of an IoT gateway is that it can provide additional security for the loT network and the data it transports. Because the gateway manages information moving in both directions, it can protect data moving to the cloud from leaks and loT devices from being compromised by malicious outside attacks with features such as tamper detection, encryption, hardware random number generators and crypto engines. 